\title{
Multi-agent Environment for Dynamic Transport Planning and Scheduling
}

\author{
Jaroslaw Kozlak $^{1,2}$, Jean-Charles Créput ${ }^{1}$, Vincent Hilaire ${ }^{1}$, and Abder Koukam ${ }^{1}$ \\ ${ }^{1}$ UTBM Systems and Transportation Laboratory, \\ University of Technology of Belfort-Montbéliard, Belfort, France \\ ${ }^{2}$ Department of Computer Science, AGH University of Science and Technology, \\ Al. Mickiewicza 30, Kraków, Poland \\ kozlak@agh.edu.pl, \\ \{Jean-Charles.Creput,Vincent.Hilaire,Abder.Koukam\}@utbm.fr
}

\begin{abstract}
Nowadays, transport requests of the society are becoming more and more common and important. Computer systems may be a useful tool for transport companies. This paper is aimed at presenting a system which simulates the functioning of a transport company. The theoretical problem, which needs to be solved, is a dynamic Pickup and Delivery Problem with Time Windows and capacity constraints (PDPTW). The nature of the problem, its distribution and the possibility of using a lot of autonomous planning modules, predestines to apply a multi-agent approach. Multi-agent approach facilitates the introduction of elements which do not appear in classical PDPTW as company organisation, different strategies of requests acceptation by different vehicles or communication among vehicles.
\end{abstract}

Keywords: Multi-agent systems, dynamic transport planning and scheduling.

\section{Introduction}

Nowadays, the importance and commonness of transport requests in the society increases. The transport services offered are more and more advanced and better designed to meet the needs of users. Important parts of costs of many goods are transport costs. The size of the market of transport services and the sales volume and derived profits intensify a competition. Therefore, a reduction of costs, better adaptation of strategy to the demand as well as better planning and scheduling of the use of available resources are important for transport companies. Computer systems may be a useful tool for transport companies. On the one hand, they may support a rapid creation of effective transport plans and schedules. On the other, they enable simulation research leading to the correct selection of company organization, and their resources like vehicles, their capacities, and their location on the depots. We present a type of transport problem which have important practical applications: the Dynamic Pickup and Delivery Problem with Time Windows (PDPTW).

Each request is described by two locations: one for pickup and one for delivery and two time windows: the time window when a pickup operation can be started and the one when a delivery operation may be started. Both the request points: pickup and delivery places should be visited by the same vehicle in proper order. There is also a 
possibility of introducing an additional capacity constraint: each request is characterised by a load, and each vehicle has a maximum capacity which cannot be exceeded by the total load.

PDPTW is worth exploring because of its practical applications, like the transport of elderly and handicapped people, shared taxi or microbus company, sealift and airlift, school bus routing and scheduling. Additionally, PDPTW is a problem relatively weakly explored, especially in comparison to VRPTW.

The goal of this paper is to present a system which simulates the functioning of a transport company. The basic problem, which needs to be solved, is a dynamic version pf Pickup and Delivery problem with Time Windows and capacity constraints, where requests arrive during system running. The nature of the problems their distribution and possibility of applying a lot of autonomous planning modules predestines to use a multi-agent approach. Multi-agent approach facilitates to consider aspects which do not appear in classical PDPTW as company organisation, different strategies of requests acceptation by different vehicles or communication among vehicles.

The structure of the paper is as follows: Section 2 contains research overview, we put the emphasis on the heuristic methods to solve pickup and delivery problem and description of multi-agent systems for transport planning and scheduling. In Section 3, main features and advantages of our approach are given. In Section 4, the model of our system is described. Section 5 presents goals and configurations of performed experiences as well as obtained results. Section 6 concludes and presents plans of future works.

\section{Research Overview}

\subsection{Heuristic Methods of Plan and Schedule Construction}

An overview of optimal and heuristic methods of pickup and delivery problem solving may be found in [3, 4, 14]. An optimal, strict solution - because of its calculation complexity - is possible to obtain only for small size problems. Heuristic solutions for PDPTW are performed using methods like tabu search [10, 11, 15] simulated annealing [11], squeaky wheel optimization with local search [12].

Very few papers focus on dynamic aspects of PDPTW problem. Usually, the algorithms are similar to the ones used for static problems, and it is assumed that incoming of new requests interrupts the optimization function which is then restarted with a new set of requests. In [7], the authors describe a method for determining times of requests generation on the basis on Poisson distribution. Space is divided into regions, and each of them has its own probability that requests pickup or delivery points will be located on it. Basing upon these parameters, the points of pickup and delivery are determined. Times in the time windows are calculated in a way to make possible that the vehicle returns to depot before the end of the simulation time. For that purpose, the times of driving between pickup and delivery points and between delivery points and a depot are taken into consideration. In [13] authors describe REBUS system, which solves real-time dynamic PDPTW. The system was designed for transporting elderly or disabled persons in Copenhagen. 


\subsection{Multi-agent Systems}

Multi-agent approach to transport problems enables the analysis of complex, heterogeneous and dynamic systems, and focuses mostly on complex cargo shipping problems (sometimes taking into consideration transshipments and transport multimodality [2]) but some systems are also targeted at transport on demand problem [8].

In [1], a method of simulated trading was presented and applied for solving vehicle routing problem. Several processes are executed concurrently and each of them represents one route. There is also one process which manages the stock of unattached requests. The idea is based on buying and selling requests by processes representing routes. The process tries to sell requests which have bad influence especially on its cost function, and to buy these which do not increase it appreciably. A graph, representing the flow of requests between routes, is built and serves for the creation of feasible and efficient plans (trading matching).

MARS [6] is a system realized to simulate planning and scheduling for a society of shipping companies using multi-agent approach. The agents represent transportation companies and trucks. Protocol Contract Net [17] is used to assign requests to particular vehicles (company is a manager and truck-contractor). Simulated trading procedure is used for dynamic re-planning (if because of unexpected events - the traffic jams - the vehicle is not able to realize a request) or for optimization of the current solution by searching to a better one.

On the basis of experience, gathered using simulated trading and MARS, a distributed multi-agent system for shipping company planning and scheduling TeleTruck [2] was realized and practically tested. The architecture of TeleTruck is based on the concept of holonic agents. In this system, there exists different kinds of agent-components (driver, truck, trailer, container), which possesses and manages specific type of resources. This approach gives a possibility of considering different aspects of transport problem (storing, transportation, management of cargos, drivers, trucks etc.).

\section{System Aims and Methodology}

Our goal is to create a system which makes possible the simulation of transport company. Transport requests, realized by a company, should suit dynamic PDPTW with capacity constraints. It is assumed that only feasible requests are accepted for performance. If the company is not able to realize a request respecting all constraints needed, such a request should be rejected. Besides, the system should simulate the network of communication connections (routes) and manage the features of scheduling process for each vehicle. A vehicle should have its own conditions of acceptance and estimation of a request.

Agent approach makes possible the development of systems consisting of many autonomous elements which are able to create plans, choose actions for realisation in a certain way, to perform its goals. Because of different locations of vehicles and of the many independent decisions they have to take, the transport planning and scheduling problem may be considered as an example of a problem with a distributed domain, which is very suitable for multi-agent approach [5]. 
Agent approach makes possible to take into consideration autonomous, goaldriven agents, which represent a company and vehicles. Each agent-vehicle manages its route. An agent estimates a request taking into consideration if it is feasible, which payment it will obtain and what expenses it will bear. A transport network may be represented as a directed graph. Agents may be equipped with message communication modules which gives a possibility of direct interactions between vehicles and drivers.

\section{System Model}

A model of multi-agent system for transport planning is composed of the following elements:

- environment: it represents a transport network and it is implemented through a graph describing communication connections,

- several kinds of agents: customer agent - responsible for the generation of transport requests, agent-company - representing a transport company and agentvehicle - representing a vehicle of transport company, like mini-bus, bus, taxi etc.

\subsection{Specification of System Organisation}

We base our work on the RIO framework previously defined in [9]. This framework is based on three interrelated concepts : role, interaction and organization. Roles are generic behaviors. These behaviors can interact mutually according to interaction pattern. Such a pattern with groups generic behaviors and their interactions constitutes an organization. Organizations are thus descriptions of coordinated structures. Coordination occurs between roles as and when interaction takes place.

In this context, an agent is only specified as an active communicative entity which plays roles. In fact agents instantiate an organization (roles and interactions) when they exhibit behaviors defined by the organization's roles and when they interact following the organization interactions. An agent may instantiate dynamically one or more roles and a role may be instantiated by one or more agents.

The organization of the MAS is described in Fig. 1. There are two organizations: one specifying the Contract Net protocol and one specifying the interactions between clients and pick-up and delivery service providers. The latter organization specifies

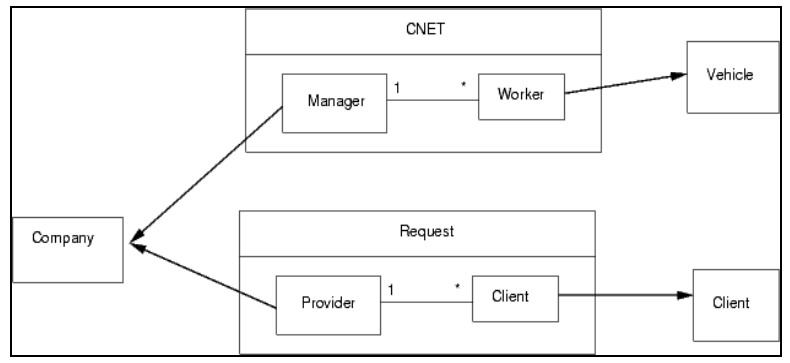

Fig. 1. System organization 
two roles : Provider and Client. The Client role represents virtual clients that send request of pick-up and delivery to a virtual Provider that accept these requests and answer with offers. The Provider role is played by the Company agent. The former organization is composed of two roles : Manager and Worker. This organization specifies a contract net based negotiation between a Manager which propose pick-up and delivery requests and some Worker which are able to realize these requests. The Manager role is played by the Company agent and Worker role are played by Vehicle agents.

\subsection{Model Elements Description}

Environment. The transport network is represented as a directed graph $T N(N, E)$, where $N$ is a set of nodes and $E$ is a set of arcs, respectively. Nodes represent the locations. They may be either a start or destination point of the goods transfer. With each node $V_{i}$ are associated the following parameters: a pair $\left(x_{i}, y_{i}\right)$, where $x_{i}$, and $y_{i}$ are coordinates on the map, numerical value describing the probability that this node will appear in the transport request as pickup or delivery point $\left(w_{i}\right)$, list of requests waiting for pickup from this node $\left(p l_{i}\right)$. Each arc $E_{i}$ is described by time period $t p_{i}$, which informs how much time the vehicle needs to traverse it. Value $t p_{i}$ expresses also the cost of travel carried out by a vehicle.

The following types of agents exist in the system: agent-customer, agent-vehicle, agent-company.

Agent-customer. Agent-customer is responsible for generation of random events. The methods of events generation is inspired by [7]. Agent Customer sends them later to agent-company. Agent Customer $A C$ is described by a list of periods of requests generations $\left(\mathrm{prg}_{i}\right)$, each period is characterized by: $\gamma_{i}$ - coefficient of Poisson distribution describing the frequency of request generation, $\beta_{i}$ - probability that time window of pickup starts just after request arrival, $\delta-$ an expected size of time windows.

Agent-company. Agent-company - is responsible for request reception from agentcustomer and its scheduling to agent-vehicles. Agent-company $A C_{i}$ is represented as a tuple ( $g, c, s, f, R L)$, where: $g$ - gains (incomes-costs), $c$ - costs, $s$ - an average level of satisfaction of clients, which should be guaranteed, $f-$ a function of choice of agent-vehicle, which will perform the request, $R L-$ a list of received requests with information concerning the state of their realization (received, rejected, scheduled to agent, pickup performed, delivery performed).

Agent-vehicle. Agent-vehicle represents vehicle. It moves among nodes in the transport network and possesses plans of its routes and schedules of request realization. To obtain realization of request, it sends offers to agent-company. An Agent-vehicle $A V_{i}$ is defined as a tuple $\left(l o c_{i}, g_{i}, c_{i}\right.$, cap $_{i}$, pass $\left._{i}, L R N_{i}, R_{i}, L O_{i}\right)$, where: $l o c_{i}$ - current location (node, direction, percentage of traversed arc), $g_{i}$ - current gain, $c_{i}$ - current costs, cap $_{i}$ - maximal capacity, pass $_{\mathrm{i}}$ - number of passengers, $L R N_{i}$ - list of request nodes, $R_{i}$ - list of nodes (route), $L O_{i}$ - list of embarked orders (after pickup and before delivery). 
Agents-vehicles plan their routes so as to visit all nodes of accepted transport requests. Each agents-vehicle uses two forms of route representation: list of request nodes and list of nodes. The list of request nodes is composed of pickup and delivery points for each accepted request ordered according to vehicle time of visit (thus each request is represented in the list by two points. On the basis of request nodes, the route composed of all traversed nodes (list of nodes) is constructed

\section{Experiments}

The goal of performed experiments is the choice of optimum company features (number of vehicles, capacity of vehicles) for the given topology of communication network as well as the quantity and features of requests. In particular, we will examine configurations with different time windows size and request frequencies.

In the experiments performed, the transport network consisting of 25 nodes and 60 arcs were used. Average length of arc is 7 minutes and 25 seconds.

Quantities of vehicles, their capacities as well as sizes of time windows were changing during experiments. The total simulation time was divided into 5 intervals (4 simulation hours each ) with different frequency of request arrival. Their Poison distribution $\lambda$ parameters were equal to $0.55,0.70,0.1,0.4,0.1$, as in one of the experiments described in [7], and the sixth supplementary period of 1 hour, aimed at allowing the realization of the last requests. The average value of generated requests was equal to 360 .

The following diagrams present the percentages of performed requests in relation to the quantity of the vehicles used and their capacities. In Fig. 2a are shown the results obtained for wide time windows (end point is calculated using even distribution, when the last possible time is the simulation end), whereas the ones presented in Fig.2b. concern narrow ones (equal 10 minutes each).

Basing upon Fig. 2a one can notice that an increase of rate of request realization of vehicle is relatively small. For this configuration of requests and transport networks, the vehicles rarely have a total load higher then 50, a stronger constraint is the

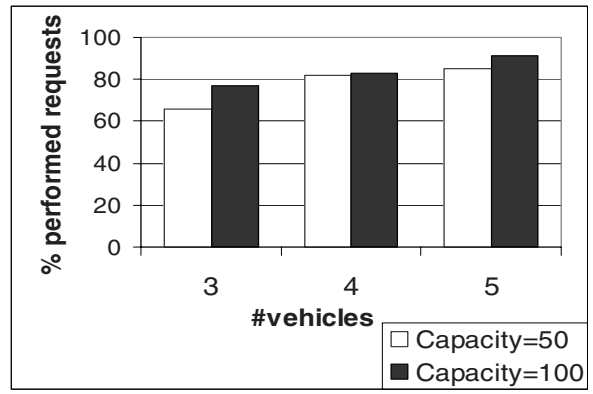

a)

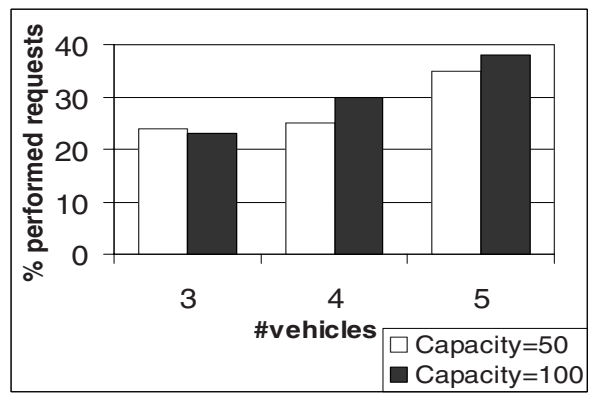

b)

Fig. 2. Request realization in relation with vehicles quantity and capacity for a problem with a) wide time windows, b) thin time windows 
participation of a vehicle in the realization of the requests previously submitted. In the case of wide time window, the increase in the quantity of vehicles improves the percentage of the performed requests.

For narrow time windows (Fig2b), the percentage of the request performed for the same number of vehicles and their capacities, is clearly lower then for wide ones. For the number of vehicles in the period of values 3-6, the difference is equal to 2-3. Similarly as in the case of wide windows, increase of the percentage of performed requests when the maximal capacity of vehicles is two times higher, is relatively small.

\section{Conclusions}

In this paper we have presented a simulation environment to solve dynamic transport problems of type PDPTW. It seems to us that the main advantages of the proposed approach to the construction of systems to transport planning is the possibility of adding emergencies, flexibility of configuration and change of features of particular vehicles, as well as taking the structure of transport organization and its policy into consideration during the optimization process.

We intend to enrich the multi-agent environment by introducing the following elements to the system, which may consist of taking into consideration several cooperating transport companies, allowing transport requests to arrive from different sources, also directly to a vehicle which is located in a node where pickup is to be realized and developing direct interactions among vehicles and request exchange between them. The another step is the implementation of the model of multi-agent systems presented in the paper by using a standard multi-agent platform, and through the development of a decentralised multi-agent system so as to improve the global computation calculation performance.

\section{References}

1. Bachem, A., Hochstattler, W. Malich, M.: Simulated Trading A New Parallel Approach for Solving Vehicle Routing Problems. Proc. of Int. Conf. "Parallel Computing: Trends and Applications", 1994

2. Burckert, H.-J., Fischer, K., Vierke., G. : Transportation scheduling with holonic MAS the TELETRUCK approach. Third International Conference on Practical Applications of Intelligent Agents and Multiagents (PAAM 98), 1998

3. Cordeau, J.-F., Laporte G.: The Dial-a-Ride Problem: Variants, Modeling Issues and Algorithms. Les Cahiers du GERAD, 2002

4. Desaulniers, G., Desrosiers, J., Erdmann, A., Solomon, M.M., Soumis, F.: The VRP with Pickup and Delivery. Les Cahiers du GERAD, 2000

5. Ferber, J.: Les systèmes multi-agents: Vers une intelligence collective, InterEditions, 1995

6. Fischer, K., Müller, J. P., Pischel, M.: Cooperative Transportation Scheduling: an Application Domain for DAI. Applied Artificial Intelligence, vol.10, 1996

7. Gendreau, A., Guertin, F. , Potvin, J.Y., Séguin, R. :Neighborhood search heuristics for a dynamic vehicle dispatching problem with pick-ups and deliveries. Rapport technique CRT-98-10, Université de Montréal (1998) 
8. Gruer, P., Hilaire, V., Kozlak, J., Koukam, A.: A multi-agent approach to modelling and simulation of transport on demand problem. J. Sołdek, L. Drobiazgiewicz (eds) : Artificial Intelligence and Security in Computing Systems, The Kluwer Int. Series In Eng. And Computer Science - 752, 2003

9. Hilaire, V.: Vers une approche de specification, de prototypage et de verification de Systèmes Multi-Agents. PhD thesis, UTBM, 2000

10. Lau H.C., Liang Z.: Pickup and Delivery with Time Windows : Algorithms and Test Case Generation. Proceeedings of $13^{\text {th }}$ IEEE International Conference on Tools with Artificial Intelligence (ICTAI'01), Dallas, USA, 2001

11. Li, H., Lim A.: A Metaheuristic for the Pickup and Delivery Problem with Time Windows. in Proceedings of $13^{\text {th }}$ IEEE International Conference on Tools with Artificial Intelligence (ICTAI'01), Dallas, USA, 2001

12. Lim, H. , Lim, A., Rodrigues, B.: Solving the Pick up and Delivery Problem using "Squeaky Wheel"' Optimization with Local Search. Proceedings of American Conference on Information Systems, AMCIS 2002, Dallas, USA

13. Madsen, O.B.G., Ravn, H.F., Rygaard, J.M.: A heuristic algorithm for a dial-a-ride problem with time windows, multiple capacities, and multiple objectives. Annals of Operations Research 60 (1995)

14. Mitrowic-Minic, S.: Pickup and Delivery Problem with Time Windows: A Survey. SFU CMPT TR. 1998-12, ftp://fas.sfu.ca/pub/cs/techreports/1998

15. Nanry, W.P., Barnes, J.W.: Solving the pickup and delivery problem with time windows using reactive tabu search. Transportation Research Part B 34, Elsevier Science Ltd 2000,

16. Potvin, J. Y., Bengio, S.: The vehicle routing problem with time windows - Part II: Genetic search., INFORMS Journal on Computing 8 (1996) 